\title{
Protocolo Protésico - Quirúrgico en Temporización Inmediata de Dientes Unitarios, Utilizando una Cubeta Multifuncional Desmontable
}

\author{
Prosthetic Protocol - Surgery in the Immediate Temporization of a Single Tooth \\ Using a Multifunctional Removable Tray
}

Leighton $\mathrm{Y}^{1}$, Carvajal $\mathrm{JC}^{2}$, Wolnitzky $\mathrm{A}^{3}$, Silva $\mathrm{R}^{4}$, Von Marttens $\mathrm{A}^{5}$

\section{RESUMEN}

Propósito: El presente reporte clínico es la descripción de un protocolo protésico - quirúrgico que permite la visualización directa del sitio operatorio y el correcto posicionamiento protésico - implantario de una corona temporal atornillada, construida inmediatamente de insertado el implante. Material y métodos: Se seleccionaron 10 pacientes del programa de especialización en implantologia bucomaxilofacial de la Universidad de Chile, cuyo criterio de inclusión principal fue ser desdentado parcial unitario posterior, con antagonista dentario natural. Como protocolo diagnóstico se solicitaron exámenes de laboratorio, exámenes radiograficos y se registraron sus arcos dentarios con impresiones de estudio a fin de obtener modelos de yeso, los que fueron posicionados en un articulador semiajustable tipo Whip Mix, donde se confeccionó un encerado diagnóstico del diente a reemplazar, libre de contacto oclusal en máxima intercuspidación (MIC) y en los movimientos excursivos mandibulares. Basado en el encerado de diagnóstico se diseño una cubeta multifuncional desmontable (U. de Chile) cuya característica fundamental es que fusiona una guía quirúrgica y una guía de montaje protésico, para una correcta temporización inmediata. El procedimiento se inicia mediante un colgajo de espesor total, posicionamiento en boca de la guía multifuncional en fase quirúrgica, para permitir el fresado del lecho implantario y la inserción implantaria. El lecho quirúrgico es suturado mediante una seda negra trensada y se confecciona en forma inmediata una corona temporal atornillada utilizando la guía multifuncional en fase de carga protésica. Resultados: Se instalaron un total de 12 implantes Renova Tapered (Lifecore) utilizando la cubeta multifuncional la cual facilitó la direción del fresado y la contrucción de sus respectivas coronas temporales de resina acrilica, no segmentadas atornilladas. Conclusiones: Con las limitaciones de este estudio, la fusión de ambos procedimientos brinda una alternativa de tratamiento segura, predecible, rápida y de fácil ejecución para el clínico, permitiendo la temporización inmediata de implantes. Reporte de técnica.

Rev. Clin. Periodoncia Implantol. Rehábil. Oral Vol. 3(1); 34-38, 2010.

Palabras clave: Cubeta multifuncional desmontable, carga inmediata.

\section{ABSTRACT}

Purpose: This clinical report is the description of a prosthetic protocol - surgery which allows direct visualization of the operative site and the correct positioning of the prosthetic-implantation of a screw retained temporary crown constructed immediately following the insertion of the implant. Methods: 10 patients, whose main inclusion criterion was having a partially edentulous posterior unit with an opposing natural tooth, were selected from the specialized program of Maxillofacial Implantology at the University of Chile. As diagnostic protocol was requested, laboratory tests, radiographic examinations and impressions of dental arches were recorded in order to obtain plaster models for the study and which were positioned in a semi-adjustable articulator type Whip Mix, which produced a diagnostic wax-up tooth replacement, free of occlusal contact in the maximal intercuspal (MIC) and mandibular excursions. Based on the diagnostic wax-up one can design a multi-functional removable tray (U. de Chile), whose key feature is that it merges a surgical guide and a prosthetic assembly guide for the correct immediate temporization. The procedure starts with a full thickness flap positioned in the mouth of the multi-functional guide of the surgical phase to allow the implant site drilling and implant insertion. The surgical site is sutured with black braided silk and you immediately draw up the screw retained temporary crown using the multi-functional guide in the prosthetic loading phase. Results: A total of 12 Renova Tapered implants (Lifecore) were installed using the multi-functional tray which provided the direction of the drilling and construction of its acrylic- resin temporary crowns without segmented screws. Conclusions: With the limitations of this study, the merging of both procedures provide a predictable safe alternative treatment that is quick and easy to implement for the clinician allowing the immediate temporization of the implant. Technical Report.

Rev. Clin. Periodoncia Implantol. Rehábil. Oral Vol. 3(1); 34-38, 2010.

Key words: Multifunctional removable tray, immediate loading.

1. Especialista en Implantología BucoMáxiloFacial Docente del programa de Especialización profesional en Rehabilitación Oral y del programa de Especialización profesional en Implantología BucoMáxiloFacial, Facultad de Odontología. Universidad de Chile. Chile.

2. Profesor Asociado del Departamento de Prótesis, Facultad de Odontología, Universidad de Chile. Director del programa de Especialización profesional en Rehabilitación Oral, Facultad de Odontología, Universidad de Chile. Director de Prótesis del programa de Especialización profesional en Implantología BucoMáxiloFacial, Facultad de Odontología. Universidad de Chile. Chile.

3. Especialista en Implantología BucoMáxiloFacial EX - Docente del programa de Especialización en Implantología BucoMáxiloFacial, Facultad de Odontología. Universidad de Chile. Chile.

4. Especialista en Implantología BucoMáxiloFacial EX - Docente del programa de Especialización en Implantología BucoMáxiloFacial, Facultad de Odontología. Universidad de Chile. Chile.

5. Profesor Asistente, Facultad de Odontología, Universidad de Chile. Director de Cirugía del programa de Especialización profesional en Implantología BucoMáxiloFacial, Facultad de Odontología. Universidad de Chile. Chile.

Correspondencia autor: Yerko Leighton. yerkoleighton@gmail.com. Trabajo recibido el 08/04/2010. Aprobado para su publicación el 18/05/2010. 
El protocolo original del Prof. Branemark, dicta un procedimiento quirúrgico de dos etapas, dejando el implante sumergido bajo la encia sin recibir cargas por un tiempo variable que fluctúa entre 16 a 20 semanas, lo que permitiría obtener la reparación de los tejidos duros y blandos, asegurando el proceso de Oseointegración ${ }^{(1,2,3)}$.

Sin embargo, la ansiedad del paciente generada por los tiempos de espera para el tratamiento protésico y la necesidad de que sus dientes perdidos fueran sustituidos a la brevedad, incentivaron a dentistas y casas comerciales a desarrollar nuevas modalidades de tratamientos protésicos implanto asistidos.

El mayor conocimiento que se tiene en la actualidad sobre la fisiología ósea y sobre los procesos reparativos de los tejidos duros y blandos, unido al gran desarrollo tecnológico que ha optimizado los sistemas implantarios en lo referente al diseño, tanto macro como micro(4), generaron nuevas líneas de investigación que modificaron los protocolos quirúrgicos y protésicos originales del Prof. Branemark, obteniendo en implantes sometidos a carga protésica inmediata o temporización inmediata, similares tasas de éxito implantario y protésico que en los procedimientos de carga convencional| ${ }^{(5,6)}$.

Todo esto ha sido el sustento que ha llevado a la Implantología a desarrollar nuevas modalidades de tratamientos protésicos como es la Carga Oclusal Inmediata en los pacientes desdentados completos, o de Provisionalización Inmediata en los casos de pacientes desdentados parciales. Estas modalidades de tratamiento protésico implanto asistido posibilita que los pacientes repongan sus dientes perdidos en un menor tiempo clínico, disminuyendo la ansiedad y mejorando su auto estima.

El éxito de la modalidad de tratamiento que contempla la temporizacion o estetica inmediata, radica en la minimización de los micromovimientos intraoseos que puedan afectar al implante, durante el período de reparación ósea, logrados al obtener un valor de Torque de Inserción implantario superior a $35 \mathrm{Ncm}$., sumado de preferencia a las caracteristicas de un diseño implantario cónico de conexión interna, con tratamiento de superficie de 3ra y 4 ta generación y por otra parte, a la protección de la estructura protésica temporal de la oclusion directa, gracias a la presencia de dientes vecinos en la arcada dentaria ${ }^{(7,8)}$.

La opción de temporizar un implante inmediatamente de insertado en el lecho óseo, es sin duda un tratamiento de elección en la actualidad, pero requiere una cuidadosa y prudente planificación con el fin de obtener un tratamiento altamente exitoso, donde la concepción y práctica de una implantologia protésicamente guiada es fundamental ${ }^{(9,10,11)}$

Los protocolos descritos para temporizar un diente unitario son las indexación, la confección directa de la corona sobre un pilar protésico y su cementación imediata, o bien, la toma de impresión inmediata a la cirugia para transferir la plataforma implantaria o el pilar protésico y la confección de una corona temporal por parte del laboratorio dental.

El presente reporte clínico diseñó un protocolo protésico/ quirúrgico que representa la fusión de una guía multifuncional unitaria que facilitará la correcta cirugia de inserción de implante y una correcta rehabilitación protésica temporal inmediata.

\section{MATERIAL Y MÉTODOS}

El estudio fue realizado en la Escuela de Graduados de la Facultad de Odontología de la Universidad de Chile, con 10 pacientes seleccionados que consultaron por libre demanda en el Programa de Especialización en Implantología Buco Máxilo Facial. Una vez reclutados los pacientes, se les instruyó respecto de las condiciones del estudio y se enrolaron todos aquellos sujetos que cumplieron con los criterios de inclusión, previa información del tipo y modalidad de tratamiento que recibirían, mediante la firma de un consentimiento informado, el cual fue aprovado por el comité de ética institucional de la Facultad de odontologia de la Universidad de Chile.

\section{Criterios de inclusión}

1. Mayor de 18 años.

2. No presentar alteraciones sistémicas que contraindiquen una cirugía de implantes.

3. Segmento unitario desdentado con hueso cicatrizado independiente del lado de la arcada con antagonista dentario.

4. Disponibilidad ósea para la inserción de un implante de 3.75 por $10.0 \mathrm{~mm}$., como mínimo.

5. Ausencia de compromiso estético por pérdida de encía

\section{Criterios de exclusión}

1. Presencia de enfermedad periodontal.

2. Presencia del hábito de fumar.

3. Presencia de Diabetes descontrolada

Los pacientes fueron examinados y se les solicitó la siguiente secuencia de examenes complementarios:

1. Fotografías Clínicas.

2. Articulación de modelos.

4. Encerado diagnóstico del sitio a tratar.

3. Radiografía Periapical de sitio a intervenir.

4. Radiografia Panorámica.

5. Tomografía Hipocicloidal de la zona.

El implante seleccionado para el estudio fue Renova Tapered (Lifecore) con superficie RBM y una conexión interna de 6 lados junto con un pilar temporal de titanio recto para confeccionar la corona temporal atornillada directa al implante.

\section{Procedimiento Prequirúrgico}

Una vez articulados los modelos de estudio en un articulador semi ajustable tipo Whip Mix, se procede a la construción de la Cubeta Multifuncional Desmontable, siguiendo los siguientes pasos:

1. Confección de un encerado diagnóstico del diente faltante, libre de contactos oclusales en máxima intercuspidación (MIC) y en los movimientos excursivos mandibulares.

2. Impresión con material elástico (hidrocoloide irreversible, Xantalgin) del modelo de yeso con el diente faltante encerado.

3. Polimerización del diente encerado para la obtención de una corona acrílica del color dentario requerido por el paciente.

4. Obtención de un nuevo modelo de yeso de arco completo

5. Realización de un estampado plástico al vacío en lámina dura.

6. Reposicionamiento de la corona temporal sobre el modelo del encerado

7. Reposicionamiento del estampado plástico confeccionado sobre el modelo de yeso que contiene la corona

8. Registro con pintura indeleble en el estampado transparente, a modo de guías de trayectoria de fresado, de los ejes mayores de las dientes vecinos a la zona a reconstruir y de la dirección del implante en el diente faltante.

9. Confección de la guía protésica quirúrgica perforando por oclusal el estampado plástico y la corona temporal, siguiendo el eje coronario visualizado en la imagen radiológica.

10. Perforación completa del provisorio en su cara oclusal, para permitir el paso del pilar protésico implantario.

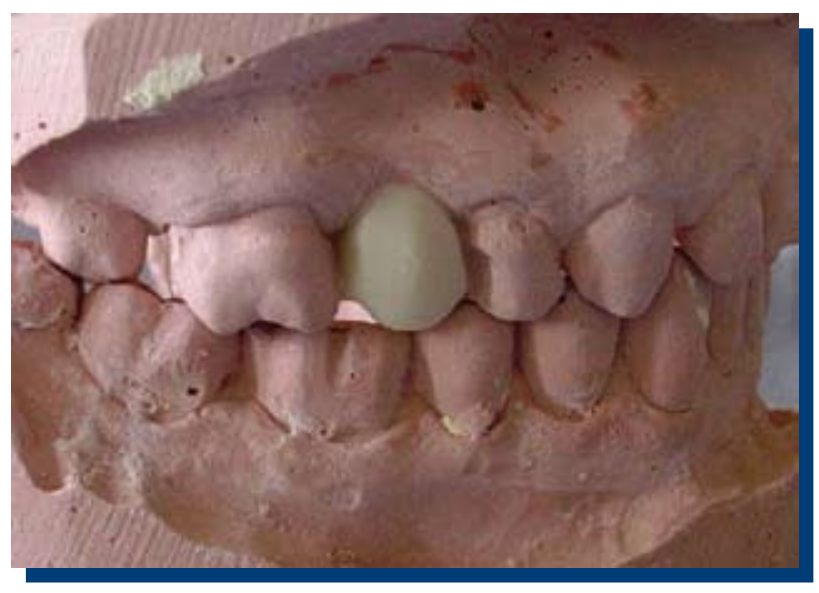

Figura 1. Encerado sin contactos. aderida. 


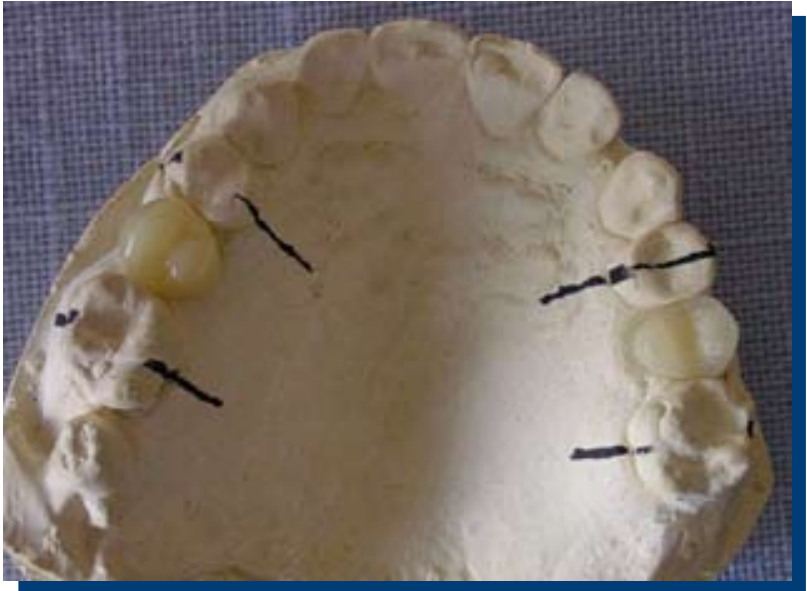

Figura 2. Polimerización de coronas en acrílico.

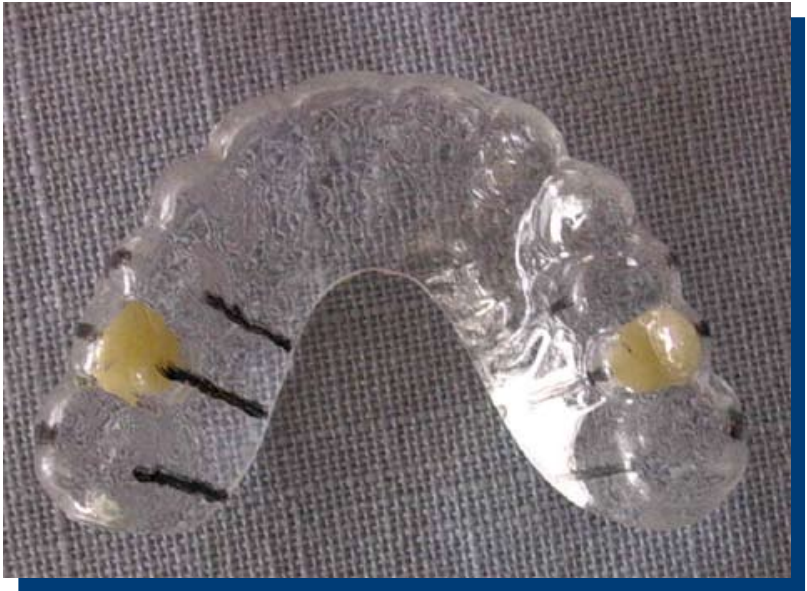

Figura 3. Montaje de provisorios sobre estampado transparente rígido, para conformar la cubeta multifunción desmontable.

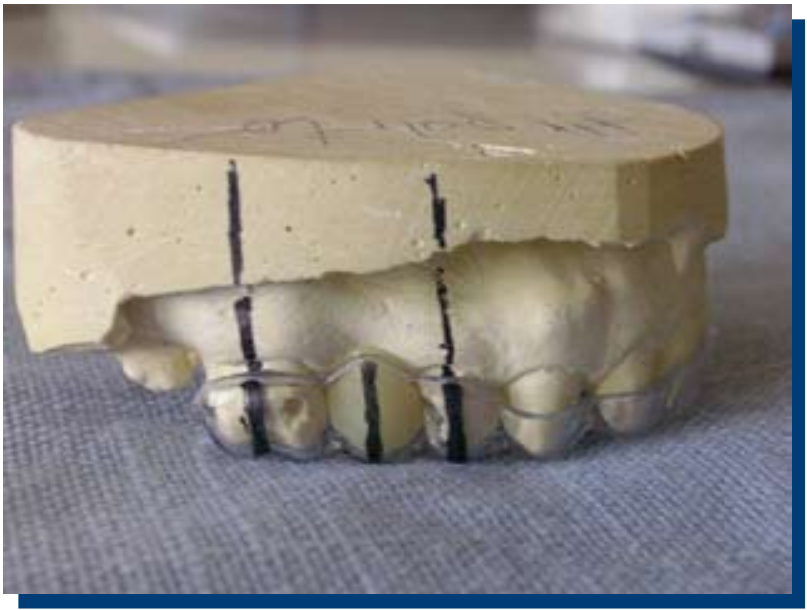

Figura 4.Trayectoria del implante y dientes vecinos.

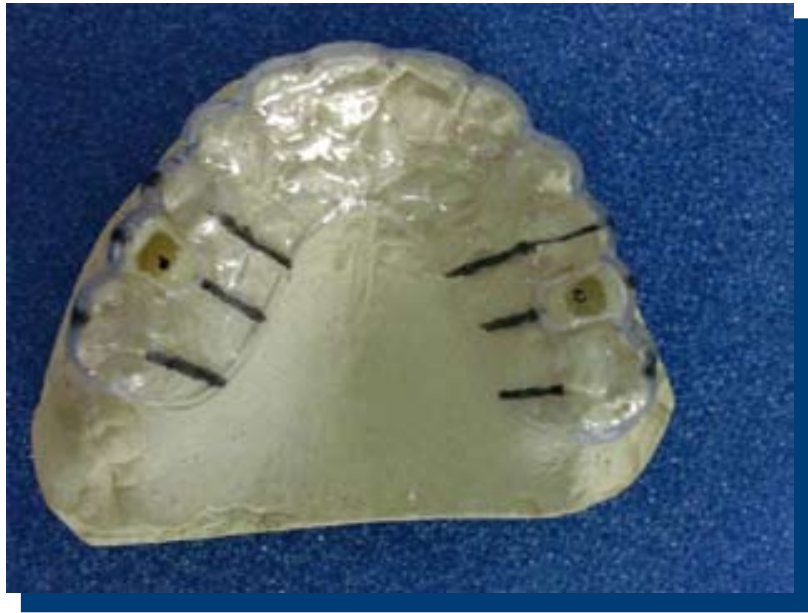

Figura 5. Guía multifuncional desmontable terminada.

El protocolo fue evaluado en su fase quirúrgica y protésica a través de una valoración dicotómica de: Óptimo o Deficiente.

\section{Valoración Fase Quirúrgica}

\section{Asentamiento de la guia multifuncional}

Se valora el asentamiento de la guía a la dentición remanente, el que debe debe ser total, sin básculas y permanecer en boca sin necesidad de sujeción manual, el incumplimiento determina la suspensión de los procedimientos (valoración pre-quirúrgica).

\section{Visualización quirúrgica}

Se valora la visión directa del lecho óseo, el acceso a los implementos quirúrgicos y la irrigación directa de la fresa. La ausencia de estas condiciónes será valorada como deficiente.

\section{Valoración de la guía en su direción de fresado óseo}

La dirección de fresado se valora por el paralelismo que debe presentar el fresado óseo, con las quías direccionales trazadas en la cubeta multifuncional, custodiando la dirección de fresado e inserción implantaria, pre-fijadas en la guía por marcas indelebles. La ausencia de esta condición será valorada como deficiente.

\section{Valoración de la Fase Protésica}

\section{Retención de la corona temporal}

Se valora que exista retención de la corona temporal a la guía protésica, con un solo eje de inserción, sin básculas y sin necesidad de sujeción manual. La ausencia de esta condición será valorada como deficiente.

\section{Valoración del posicionamiento implantario y su relación protésica}

Se valora el posicionamiento del pilar protésico al implante, el cual debe realizarse a través de la corona temporal, por el centro de la cara oclusal. La ausencia de esta condición será valorada como deficiente.

3. Valoración del asentamiento de la corona protésica terminada y su conexión al implante

La cubeta debe permitir el posicionamieto en íntimo contacto de la corona terminada a la plataforma implantaria, condición clínica que será valorada por la ausencia de penetración de la sonda de caries, ratificado en la imagen radiológica por la ausencia de luz implantaria, 
en la zona de sellado protésico. La ausencia de esta condición será valorada como deficiente.

\section{Tiempo de confeción de corona protésica temporal}

Cuantifica el tiempo empleado en la confección de la corona temporal.

\section{Procedimiento Quirúrgico}

Las cirugías se realizaron el los pabellones quirúrgicos de la Escuela de Graduados de la Universidad de Chile, administrando a los pacientes una dosis profiláctica de antibioterapia con Amoxicilina de 2 gramos una hora antes de la cirugía. También se administraron analgésicos antiinflamatorios no-esteroidales, Meloxicam $7.5 \mathrm{mg}$ cada 12 hrs., y Paracetamol $500 \mathrm{mg}$ cada 8 hrs por tres días, a partir del día de la cirugía. Se solicitó realizar enjuagatorios de Perio Aid mantención, 2 veces al día por 10 dias, a partir del día anterior a la cirugía.

El procedimiento quirúrgico consistió en la elevación de un colgajo de espesor total, con una insición supra crestal y dos descargas laterales para la exposición del reborde alveolar. Una vez visualizado el lecho quirúrgico se realiza el posicionamiento de la cubeta multifuncional para ser utillizada en su rol de guia en fase quirúrgica, evaluando su estabilidad, visión quirurgica y acceso de la instrumentación a fin de iniciar el fresado óseo, verificando la dirección de fresado por las marcas indelebles efectuadas en el procedimiento de confección de la guía en el laboratorio. Terminada la secuencia de fresado óseo e inserción implantaria, el acceso quirúrgico es suturado mediante una seda negra trensada, sin tensión.

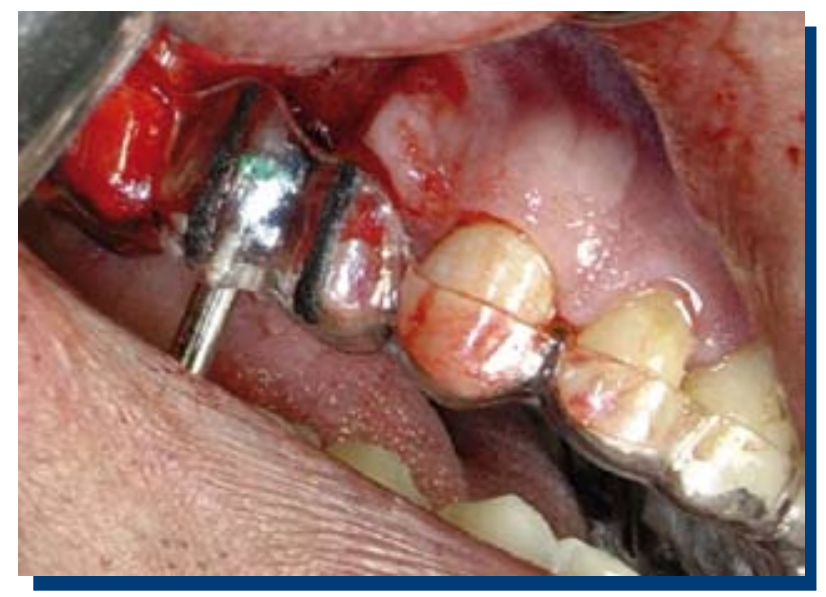

Figura 6. Guía multifuncional pick-up en fase quirúrgica evaluando su estabilidad, visión quirúrgica y determinado la posición tridimensional del implante orientada por las marcas.

\section{Fase Protésica}

Insertado el implante, se conecta un pilar protésico recto de titanio seccionándolo a la altura requerida por la oclusión dentaria, se posiciona la cubeta multifuncional que contiene la corona temporal, verificando que esta se encuentre lo suficientemente espaciada en su interior para que no impida el paso del pilar protésico a través de ella, ni el correcto posicionamiento de la cubeta en boca. Se sella la entrada del pilar protésico con teflón para impedir que ingresen a su interior residuos y se procede a rellenar con resina acrílica fluida de autopolimerización (Alike, GC America INC) el espacio vacío entre la pared del pilar y la pared interna del la corona, con el fin de unir ambas estructuras. Finalizado el período de polimerización de la resina acrílica, se suelta el tornillo de unión del pilar protésico al implante y se desmonta la cubeta multifuncional de boca, permaneciendo en ella posicionada, la corona temporal junto al pilar protésico unido con acrílico. Se afinan los márgenes fuera de boca dando los perfiles de emergencia dentarios al temporal y suavizando sus contornos. Afinada y pulida la corona provisoria, se posiciona en la guía multifuncional corroborando su eje de inserción, para ser llevada en la corecta posición protésica y ser atornillada al implante; se procede a sellar la entrada al tornillo protésico respetando la inclinación cuspidea diseñada.
Terminado el procedimiento se procedio a la toma de una radiografia periapical de control y a entregar las indicaciones post operatorias quedando las coronas protésicas temporales libres de contacto dentario en MIC y movimientos excursivos mandibulares.

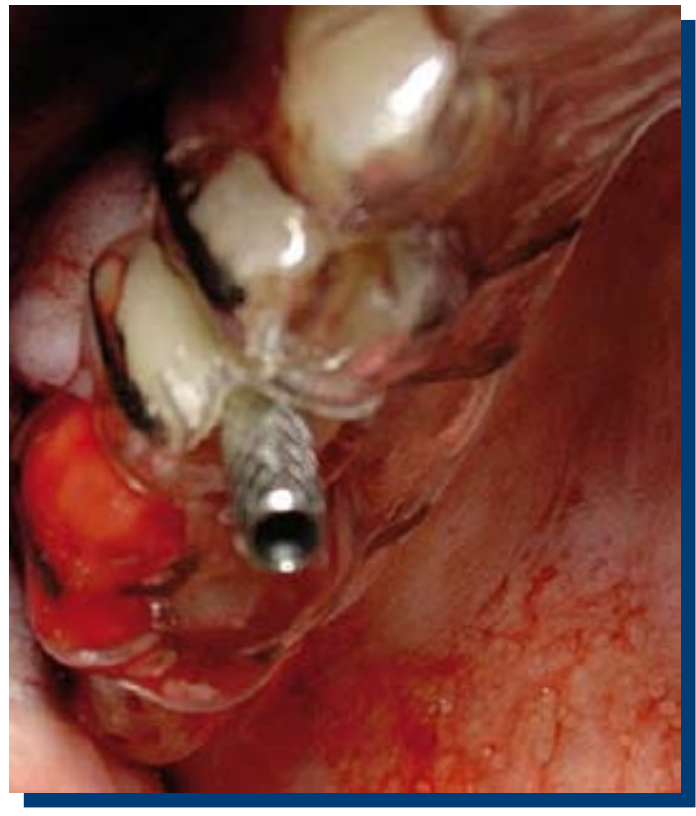

Figura 7. Guía multifuncional en fase protésica verificando la altura del pilar temporal de titanio, su ausencia de contacto y tensión con el provisorio.

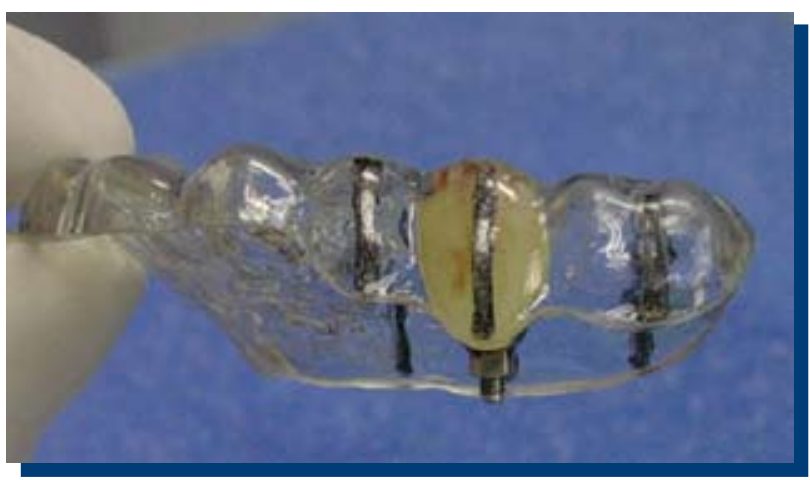

Figura 8. Provisorio soldado mediante acrílico al pilar protésico recién retirado de boca y muy bien montado en la cubeta multifuncional en fase protésica.

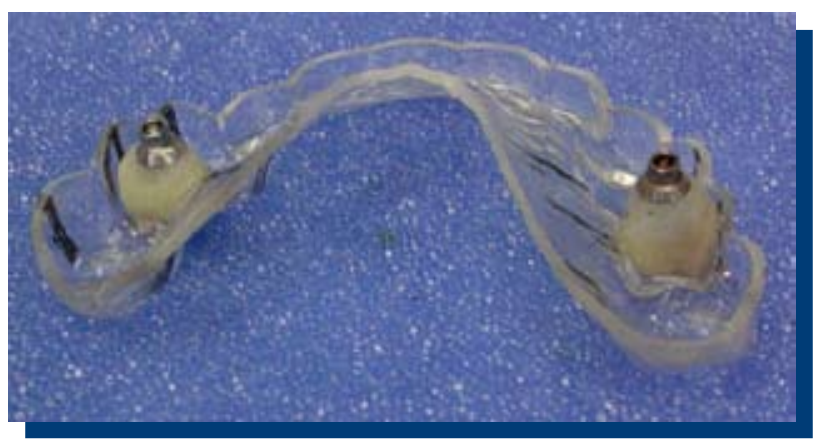

Figura 9. Provisorio rebasado, afinado y montado en la cubeta multifuncional en fase protésica, listo para su inserción en boca respetado su espacio protésico diseñado en la fase de laboratorio. 


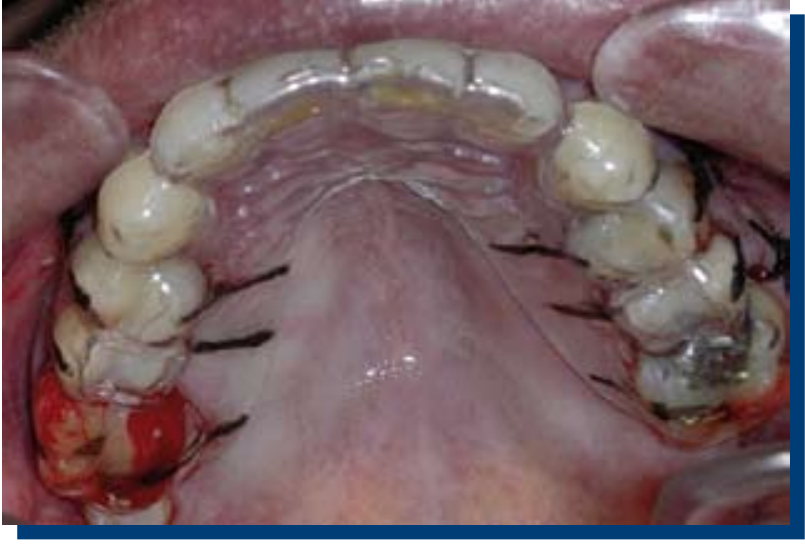

Figura 10. Provisorio atornillado junto a la guía multifuncional, obliterado sus perforaciones de acceso al pilar protésico mediante resina acrílica y asegurando el óptimo posicionamiento coronario.

\section{RESULTADOS}

Se trataron 10 pacientes instalando un total de 12 implantes, logrando un torque de inserción quirúrgico promedio de 44.7 newton. No se registraron complicaciones quirurgicas como dehisencias o fenestraciones óseas y se instalaron un total de 12 prótesis fijas unitarias temporales, confeccionadas con el método descrito. El tiempo de trabajo clínico para el procedimiento protesico fue en promedio 31 minutos.

Tabla 1. Describe los criterios de éxito quirúrgicos de los 12 casos realizados.

\begin{tabular}{|l|c|c|c|}
\hline \multicolumn{4}{|c|}{ Criterio de Exito Fase Quirúrgica } \\
\hline & Optimo & Deficiente & Total casos \\
\hline Asentamiento Guía Quirúrgica & 12 & - & 12 \\
\hline Visualización Quirúrgica & 12 & - & 12 \\
\hline Valoración en Dirección de Fresado & 11 & 1 & 12 \\
\hline
\end{tabular}

Tabla 2. Describe los criterios de éxito Protésicos de los 12 casos realizados.

\begin{tabular}{|l|c|c|c|}
\hline \multicolumn{5}{|c|}{ Criterio de Exito Fase Protésica } \\
\hline & Optimo & Deficiente & Total casos \\
\hline Retención Corona Temporal & 12 & - & 12 \\
\hline $\begin{array}{l}\text { Valoración del posicionamiento } \\
\text { implantario y relación protésica }\end{array}$ & 11 & 1 & 12 \\
\hline $\begin{array}{l}\text { Valoración del asentamiento de } \\
\text { la corona protésica terminada y } \\
\text { su conexión al implante }\end{array}$ & 12 & - & 12 \\
\hline
\end{tabular}

En forma no programada se obtuvo una ganancia estética al obtener conformación de papilas dentarias debido a los contornos dentarios de la corona temporal, diseñados a partir del encerado diagnóstico, permitiendo una coexistencia en salud entre las estructura protesicas y los tejidos blandos periimplantares.

\section{DISCUSIÓN}

Las modalidades de confección de coronas temporales en implantologia oral frecuentemente utilizadas en la práctica clínica, consideran la toma de impresión directa post quirurgica , la indexación o bien la confección directa de la corona temporal sobre el pilar protesico, pero ninguna de las modalidades descritas fusionan las dos actos terapéuticos, protesico y quirurgico, donde la inserción quirurgica de un implante es facilitada con el uso de una Guía Quirúrgica que oriente espacialmente al cirujano, proporcionandole una guia de fresado y a su vez, esta misma guia podria contribuir a la confeccion de un diente temporal como Guía Protésica, ahorrando tiempo y posicionandolo en el espacio protesico requerido.

La Guía Multifuncional Desmontable U. de Chile optmiza todo el procedimiento terapéutico asegurando el posicionamiento implantario y la ubicación de su corona clinica.

\section{CONCLUSIÓN}

La guía quirúrgica-protésica multifuncional desmontable permite la visualización directa del acto quirúrgico y el correcto posicionamiento coronario de una corona temporal inmediata, atornillada directamente al implante brindando una alternativa de tratamiento segura, predecible y de fácil ejecución para el clínico, junto con cumplir las exigencias estéticas y de comodidad para los pacientes.

\section{REFERENCIAS BIBLIOGRÁFICAS}

1. Brånemark P-I, Hansson BO, Adell R, et al.Osseointegratedimplants in the treatment of the edentulous jaw. Experience from a 10-year period. Scand J Plast Reconstr Surg1977;16:1-132.

2. Adell R, Lekholm U, Rockler B, Brånemark P-I. A 15-year study of ossseointegrated implants in the treatment of the edentulous jaw.Int $\mathrm{J}$ Oral Surg 1981;10:387-416.

3. Albrektsson T, Hansson T, Lekholm U. Osseointegrated dental implants. Dent Clin North Am 1986;30:151-174.

4. Deporter DA, Watson PA, Pilliar RM, et al. A histological assessment of the initial healing response adjacent to porous surfaced Ti alloy dental implants in dogs J Dent Res 1986;65:1064-1070.

5. Rocci A,Martignoni M, Gottlow J, Rangert B. Immediate function of single and partial reconstructions in the maxilla using MK IV fixtures.A retrospective analysis.Appl OsseointegrationRes 2001;2:22-26.

6. Hui E, Chow J, Li D, Liu J, Wat P, Law H. Immediate provisional for single-tooth implant replacement with Brånemark System: Preliminary report. Clin Implant Dent Relat Res 2001;3:79-86.
7. Szmukler-Moncler S, Salama H, Reingewirtz Y, Dubruille JH.Timing of loading and effect of micromotion on bone-dental implant interface: A review of experimental literature. J Biomed Mater Res (Appl Biomater) 1998;43(2):192-203.

8. Szmukler-Moncler S, PiattelliA, Favero GA, Dubruille JH.Considerations preliminary to the application of early and immediate loading protocols in dental implantology. Clin Oral Impl Res 2000;11(1):12-25.

9. Kan JYK, Rungcharassaeng K. Immediate placementand provisionalization of maxillary anterior single implants: A surgical and prosthodontic rationale. Pract Periodontics Aesthet Dent 2000;12(9):817-824.

10. Kawahara $\mathrm{H}$, Kawahara D, Hayakawa M, et al. Osseointegration under immediate loading: biomechanical stress-strain and bone formationresorption. Implant Dent 2003;12(1):61-68.

11. Tiziano Testori, Francesca Bianchi, Massimo Del Fabbro, Serge Szmukler-Moncler, Luca Francetti, Roberto Ludovico Weinstein Immediate non-occlusal loading vs.early loading in partially edentulous patients Pract Proced Aesthet Dent 2003;15(10):787-794. 